\section{- Análisis de Estrategias \\ Competitivas en Sectores Industriales del Perú}

* Claudio Gonzales M.

* Luz Espilco B.

* Elvia Aragón L.

\section{RESUMEN}

El artículo presenta las estrategias competitivas planteadas por Michael Porter, para que una empresa pueda mantenerse y/o expandirse dentro de un sector industrial en un mercado de libre competencia. El análisis se orienta a la realidad peruana.

Palabras Claves: Economías de escala. Diferenciación. Segmentación de mercados.

\section{ABSTRACT}

This article shows the competitive strategies stated by Michel Porter to keep and/or widen an enterprise within an industrial sector in a free-competence market. Such an analysis focuses on peruvian reality.

Key Words: Scale economy models. Differentiation. Market division.

\section{INTRODUCCIÓN}

Michael Porter, estableció las estrategias básicas que una empresa puede emplear para lograr ser competitiva en su entorno. Estas estrategias genéricas son: liderazgo en costos, diferenciación y enfoque 0 alta segmentación.

La estrategia de liderazgo en costos fue muy popular en la década de los años 70 , debido al concepto muy arraigado de la curva de experiencia y al objetivo de mantener el costo más bajo frente a los competidores y lograr un volumen alto de producción.

La estrategia de diferenciación era la de crear un producto o servicio que fuera percibido en toda la industria como único. La diferenciación se consideraba como la barrera protectora contra las 5 fuerzas competitivas.

Finalmente, la estrategia de enfoque o alta segmentación, consistía en concentrarse en un grupo específico de clientes, en un segmento de la línea de productos o en un mercado geográfico.

\section{LAS 5 FUERZAS COMPETITIVAS}

Porter plantea 5 fuerzas competitivas que pueden afectar las operaciones/estabilidad de una empresa las cuales son:

- Fuerza de los clientes

- Fuerza de los proveedores

- Fuerza de los potenciales competidores (nuevos competidores)

- Fuerza de los actuales competidores

- Fuerza de los productos sustitutos

Estas fuerzas influyen en las empresas del siguiente modo:

1. Los clientes: pueden ejercer una presión sobre las empresas para reducir el precio del producto, afectando sus márgenes de beneficio.

2. Los proveedores: pueden ejercer una presión sobre las empresas para aumentar el precio de los insumos, encareciendo el producto de la empresa.

* Estudiantes de la Maestría en Ingeniería Industrial

Facultad de Ingeniería Industrial, UNMSM

E-mail: cgonzales@cajamarquilla.com.pe 


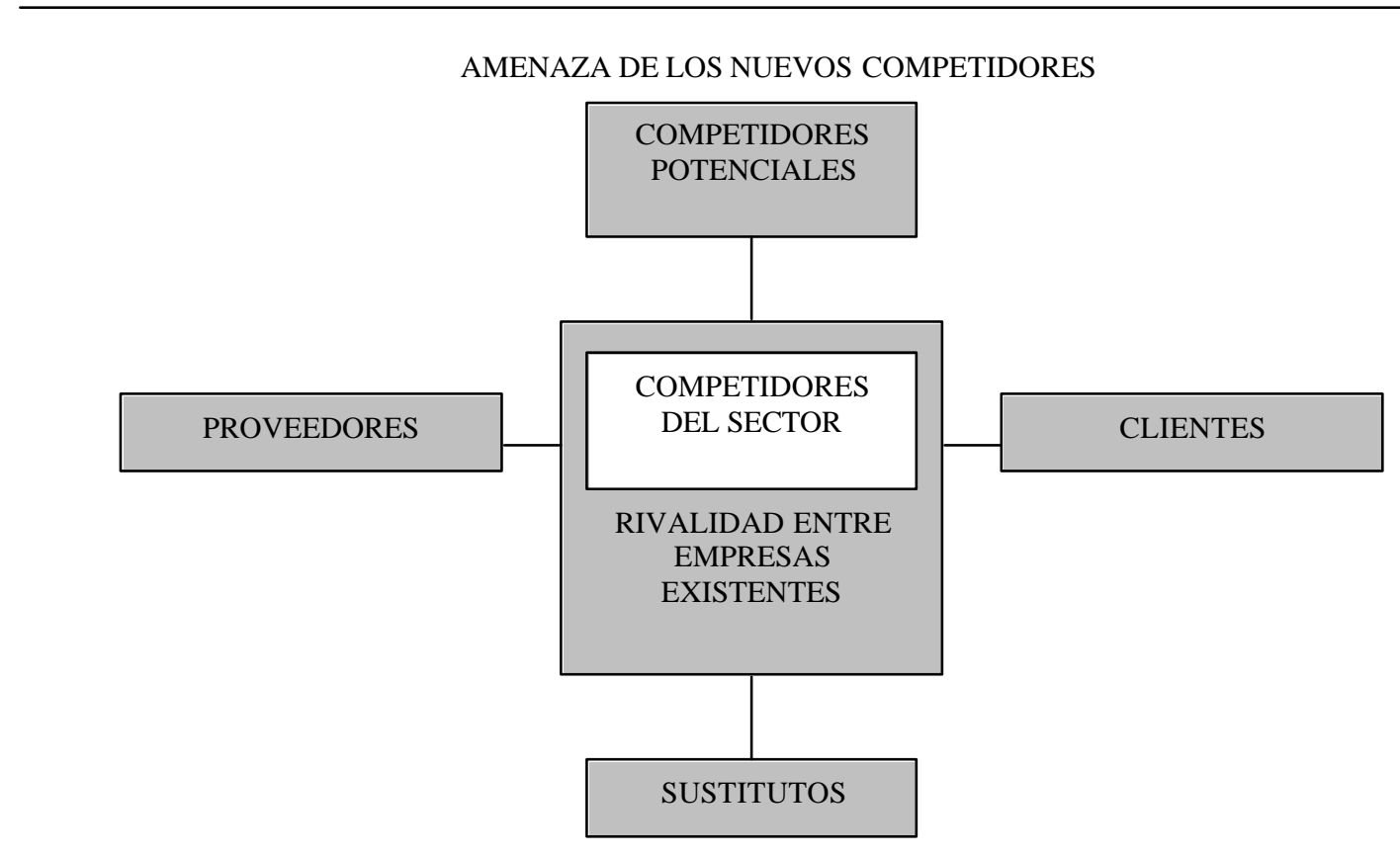

Figura 1. Modelo de las 5 fuerzas de Porter

3. Los potenciales competidores: pueden ingresar al mercado y tomar posesión de parte del mercado ya sea reduciendo precios o diferenciándose.

4. Los actuales competidores: pueden aumentar su participación en el mercado lanzando nuevos productos, mejorando el servicio, reduciendo sus precios, etc.

5. Los productos sustitutos: pueden reducir la participación del mercado de las empresas, por que pueden ser mas baratos y con el tiempo podrían apoderarse del mercado total.

\section{ESTRATEGIAS GENÉRICAS CONTRALAS5FUERZAS}

\section{La Estrategia de Liderazgo en Costos}

Esta estrategia plantea lo siguiente:

- La construcción de plantas donde se puedan producir grandes volúmenes, de manera eficiente.

- Una fuerte inversión inicial en equipos de tecnología.
- La reducción de costos, como consecuencia del efecto de la curva de la experiencia. ${ }^{1}$

- Rigurosos controles de costos y gastos indirectos

- La reducción de costos en fuerza de ventas, servicios, I\&D² y publicidad.

- El diseño de productos para que sean fáciles de producir.

- La disposición de precios bajos, para lograr una alta participación de mercados (aunque inicialmente haya pérdidas).

En nuestro medio, sólo una mayor participación en el mercado permitiría lograr economías de escala y con esto las empresas lograrían bajar sus costos y como consecuencia poseen un mayor rendimiento en el sector industrial.

Cuando se presenta este escenario de "mayor participación en el mercado y bajo costo", las empresas obtienen mayores utilidades, las cuales pueden ser utilizadas para la reinversión y el mantenimiento de ser "el líder en costos".

\footnotetext{
Cuando el costo unitario de un producto homogéneo, medido en unidades monetarias constantes, disminuye en un porcentaje fijo y previsible, cada vez que la producción acumulada se duplica.

2 Investigación y desarrollo.
} 
Obviamente, sólo empresas con una mayor participación en el mercado pueden optar por esta estrategia, seguidamente se detalla el por qué:

Cuando los costos son más bajos:

- Se crea una defensa contra los clientes poderosos, ya que estos solo pueden presionar para reducir el precio al siguiente competidor que es menos eficiente.

- Se crea una defensa contra los proveedores poderosos, puesto que cuentan con mayor flexibilidad para soportar cualquier aumento del precio en los insumos.

- Se crea una defensa contra los productos sustitutos.

- Existirán rendimientos aun después que los competidores actuales no tengan utilidad, debido a la fuerte competencia.

- Las economías de escala y la curva de la experiencia, son barreras de entrada a los nuevos competidores.

Sin embargo, los riesgos de esta estrategia son:

- Los cambios tecnológicos, eliminan la ventaja obtenida por la curva de la experiencia.

- Los nuevos seguidores y nuevos competidores (con capacidad de inversión), tendrán mayor facilidad para el aprendizaje.

- El enfoque sólo en los costos, no permite ver los cambios que requiere el mercado, en las variables del marketing mix.

\section{La Estrategia de Diferenciación}

Plantea lo siguiente:

"Crear algo que sea percibido en el mercado como único", esto no significa que la empresa descuide su atención en los costos.

Algunas formas de diferenciarse son a través de:

- Diseño de productos

- Avance tecnológico

- Apariencia exterior

- Imagen de la marca

- Cadenas de distribuidores

- Servicio de postventa

Sin embargo, a veces ésta estrategia de diferenciación impide obtener una mayor participación del mercado, ya que casi siempre requiere de una percepción de exclusividad. La ventaja es que sirve como protección contra las 5 fuerzas competitivas, ya que:
- Crea una defensa contra los competidores actuales y potenciales, debido a la lealtad de los consumidores por la marca y una menor sensibilidad en el precio.

- Se obtienen márgenes más elevados, lo cual sirve para tratar con el poder de los proveedores.

- Reduce el poder del cliente, debido a que no tienen alternativas de comparación y por lo tanto son menos sensibles al precio.

- Se crea una barrera frente a los productos sustitutos debido a la lealtad del consumidor.

Para lograr lo antes mencionado, se necesita de actividades comerciales costosas tales como comunicación, empaque, canales de distribución, etc., que si se lo compara con una buena posición de costos, resultan incompatibles.

Los riesgos de esta estrategia son:

- La diferencia de costos, entre el líder y las empresas diferenciadas, resulte demasiado elevado para retener la lealtad de la marca.

- Las imitaciones limiten la diferenciación percibida.

- Disminuya en los clientes la necesidad por el factor de diferenciación.

Hay que resaltar que este tipo de estrategia es la más acertada para su aplicación en la actualidad ya que está enfocada en la satisfacción total del cliente, a través de los requerimientos que éste solicita. Siempre y cuando existan 20 más competidores de productos similares. Para lograrlo, las empresas invierten en publicidad, I\&D, nuevas tecnologías y promueven el consumo de sus productos en el mercado.

\section{La Estrategia de Enfoque o Alta Segmentación}

Esta estrategia plantea lo siguiente:

La empresa basa sus objetivos en las necesidades de un segmento del mercado, en un segmento de la línea del producto o en un mercado geográfico. Bajo la premisa de: "Se puede servir con mayor efectividad o eficacia a un objetivo estratégico o nicho, que competir de forma mas general".

Con esta estrategia se puede lograr:

- Una diferenciación o una mayor ventaja de costos 0 ambos, pero únicamente respecto al segmento elegido.

- Una mayor participación en el segmento elegido, pero una menor participación respecto al mercado total. 


\section{VENTAJA ESTRATÉGICA}

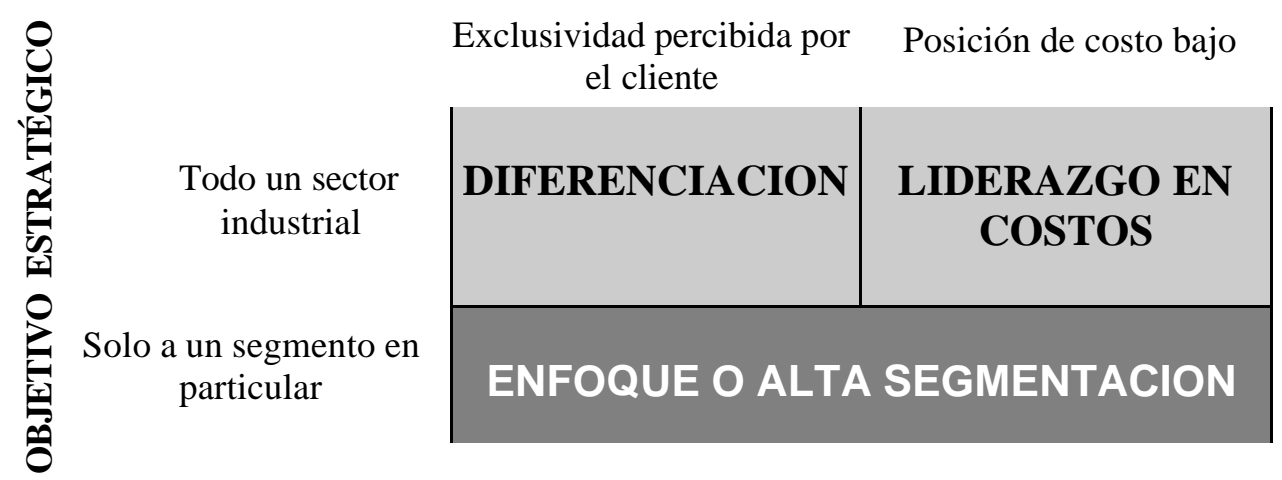

Figura 2. Estrategias genéricas de Porter

- De vez en cuando se puede obtener rendimientos mayores al promedio en el sector industrial.

\section{APLICACIÓN DE ESTRATEGIAS COMPETITIVAS EN SECTORES PERUANOS}

\section{Liderazgo en Costos}

Sector: Textil

Ubicación del Mercado: Gamarra - La Victoria (Lima) Empresas del sector: Unión de empresarios textiles de Gamarra

Amenazas de nuevos competidores: Productores textiles chinos

Los productos textiles chinos han ingresado al mercado peruano con precios reducidos inclusive hasta la mitad del precio de venta de un producto peruano. Como consecuencia la unión de empresarios textiles de Gamarra, no puede defenderse, debido a que sus costos de producción no les permiten llegar al precio de venta de los productos chinos. Por lo tanto, los chinos se convierten en líderes en costo y se apoderan del mercado.

Sector: Industria Cervecera

Ubicación del Mercado: Perú

Empresas del sector: Unión de cervecerías peruanas Backus \& Jhonston

Amenazas de nuevos competidores: Ambev
Actualmente la unión de cervecerías peruanas Backus \& Jhonston, fija el precio de la cerveza en el mercado ya que si bien es cierto es una empresa monopólica. Con el nuevo acuerdo entre Brasil y Perú la empresa brasilera Ambev fabricante de la cerveza Brahma piensa introducirla al mercado peruano a un precio de venta de la mitad de las cervezas fabricadas por Backus \& Jhonston, convirtiéndose en un líder en costos y apoderándose del mercado peruano de cervezas.

\section{Diferenciación}

Sector: Restaurantes de comida rápida Ubicación del Mercado: Lima metropolitana Empresas del sector: "Mc Donalds", "Bembos Burger Grill" y "Burger King"

Estas empresas se diferencian por el tipo de productos que ofrecen a sus clientes y que son calificados por sus atributos, entre los cuales destacan, tamaño, sabor, presentación, precio, local, marca, etc.

\section{Sector: Agroindustrial}

Rubro: Productos no tradicionales

Ubicación del Mercado: Internacional

Para poder aplicar esta estrategia a un producto agroindustrial no tradicional se debe diferenciar el producto según lo siguiente:

- El envase o empaque del producto debe tener la marca e instrucciones en el idioma del país de destino 
- Los colores del envase o empaque deben tomarse en cuenta según las tradiciones del país de destino, por ejemplo: en China los colores oscuros no son apreciados, en Japón el blanco significa luto, etc.

- Se debe elegir el canal de distribución adecuado para la venta del producto.

Entre otros factores se encuentran, los gastos por publicidad, promoción, establecimiento de la marca, etc.

\section{Enfoque o Alta Segmentación}

Está estrategia es la mas adecuada para ser empleada en nuestro país por las PYMES, ya que pueden especializarse en un sector, un ejemplo claro son los fabricantes de ropa ubicados en Gamarra, en el distrito de la victoria en Lima.

La mayoría de estos empresarios, dirigen sus productos solo a ciertos nichos del mercado, tales como "ropa para niños", "ropa para adolescentes", "ropa para adultos", "ropa para gordos", "ropa para ancianos", etc.

Debido a que el poder adquisitivo en Perú es bajísimo, la mayoría de productos de consumo masivo sería evaluado primero por el precio y segundo por calidad, pero las empresas que utilicen ésta estrategia pueden satisfacer estos dos requerimientos, de precio y calidad abasteciendo solo un nicho de mercado y dejando otros nichos a otra empresas del mismo sector.

\section{ANÁLISIS Y DISCUSIÓN}

Se ha visto tres estrategias que permiten a una empresa defenderse de las 5 fuerzas competitivas, siendo la estrategia de diferenciación y la de enfoque la más acertada para ser aplicada en nuestro medio 0 al menos en el mercado peruano.

No se puede dejar de mencionar otras estrategias vigentes de mercadotecnia tales como las de enfoque de Al Ries (Especialista, líder, mejor precio, el nombre mas apropiado para el producto), estrategias de crecimiento de Igor Ansoff (Penetración en el mercado, desarrollo del mercado, desarrollo del producto y diversificación de productos), estrategias de crecimiento de Philip Kotler (Intensivo: Penetración en el mercado, desarrollo del Mercado y desarrollo del producto; Integración: Regresiva, Progresiva y horizontal; Diversificación: Concéntrica, Horizontal y Conglomerada).
Las 12 estrategias genéricas de Ivan Allaire y Mihaela Firsirotu (Innovación y penetración de merados, diversificación, extensión de mercados, extensión de productos, liderazgo en costos, concentración, especialización, intersticios, sectores industriales no ligados y holding financiero.

Estas estrategias además de permitir la defensa contra las 5 fuerzas competitivas definidas por Porter, también permiten el ataque. La revisión de estas y otras técnicas de mercadotecnia quedan para consulta del lector.

\section{CONCLUSIONES}

Las 5 fuerzas competitivas definidas por Porter son: Proveedores, competidores dentro del sector, Clientes, Potenciales competidores y Sustitutos. Cualquier influencia de estas fuerzas sobre la empresa constituida puede afectar su existencia.

Las 3 estrategias que puede emplear una empresa constituida para que pueda defenderse de estas fuerzas competitivas son: Liderazgo en costos, diferenciación y enfoque o alta segmentación.

En la actualidad la estrategia de liderazgo en costos es aplicada para productos de consumo masivo que necesitan de poca publicidad.

La estrategia de diferenciación es la mas adecuada siempre y cuando existan 2 o más competidores, ya que una sensibilización en el costo podría hacer que los consumidores no dependan de uno u otro producto.

La estrategia de enfoque es la que debería ser usada por las Pymes, ya que como el mercado peruano es pequeño, deberían enfocarse en satisfacer ciertos sectores, tal como lo hizo el grupo Añaños con su producto Kola Real.

Bajo el escenario económico actual, el uso de estas 3 estrategias pueden ser usadas como básicas, sin embargo existen más técnicas de mercadotecnia que no solo permiten una defensa, sino también un ataque.

\section{BIBLIOGRAFÍA}

1. Hill, M. R., Alexander, S. R. y Cross, S. J. (1980). Mercadotecnia Industrial. Diana, México.

2. Healey, J.J. (1998). New Ideas in Industrial Marketing. Coram \& Hill, USA, pp.21-28. 


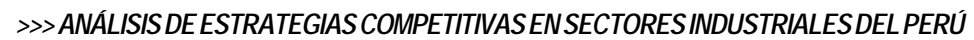

3. Kotler, Phillip; Armstorng, Gary. (1996). Mercadotecnia. Prentice Hall Hispanoamérica S.A., México, $6^{a}$ edición, pp. 700-715.

4. Lambin, Jean Jacques. (1995). Marketing estratégico. Mc Graw Hill/Interamericana de España, $3^{\mathrm{a}}$ Edición, MadridEspaña, pp. 321-358.
5. Porter, E. Michael. (1997). "Estrategia Competitiva: Técnicas para el análisis de los sectores industriales y de la competencia". Cía. Editorial Continental, México.

6. Vazquez, C. R.; Santos, V. M. L.; Sanzo, P. M.J. (1998). Estrategias de Marketing para Mercados Industriales: Producto y Distribución. Ed. Civitas S.A.,Madrid-España, pp. 65-98. 